\title{
Fluid flow modelling of a micro-valve
}

\author{
Olivier Smal ${ }^{\mathrm{a}}$, Benoît Raucent, Hervé Jeanmart
}

Department of Mechanical Engineering, Ecole Polytechnique de Louvain, Université catholique de Louvain, 2 Place du Levant, 1348 Louvain-la-Neuve, Belgium

Received 16 July 2008, Accepted 03 February 2009

\begin{abstract}
This paper presents a fluid flow analytical model of a micro check valve based on an ortho-planar spring. The comparison with a coupled fluid-structure finite-element model shows that the analytical model gives results very close to the finite-element model (FEM). These models are then validated using flow rate measurements on valve prototypes.
\end{abstract}

Key words: micro-valve; ortho-planar spring; fluid flow model

\section{Introduction}

Micro-valves are one of the most important components of micropumps which exist in hundreds of configurations [1]. They exist in various working principles and designs as presented in $[2,3,4]$. Recent advances in microtechnology are contributing to design smaller size, higher pressure valves. In order to avoid the redesign and the manufacture of several versions of the valve, it is helpful to build a model of the fluidic behavior of the microvalve. This is even more true with micro components as it is more difficult and requires accurate specialized equipment to make measurements and analysis on a small valve than on a macro one. Fluidic models are generally finite-element models (FEM) in the literature [5, 6, 7, 8] where either the deformation of the moving part is computed by FEM, or the fluid flow is computed by FEM, or both as the situation involves complex fluid-structure interaction. A few approximate analytical models have been developed but they are far from reality, as in [9] where the authors neglect the entrance and exit effects on the valve and assume that a Poiseuille flow-profile prevails over the full length of the gap.

This paper presents and compares two models, an analytical one and a FEM, for a particular micro checkvalve based on an ortho-planar spring [10]. A previous paper [11] presents a detailed analysis, including design, manufacturing and modelling of the deformation, of the spider-spring used in our valve. In the present paper, we focus on the fluid flow modelling of the valve. The analytical model presented here is very close to the FEM simulations but has the big advantage of being much less time consuming. It also allows parametric studies for design improvement.

The paper first presents the structure of the valve being analyzed. The second part presents the manufacturing of the valve components. Then, the fluid flow is described. The next section deals with the new analytical model. Then, a fluid-structure FEM of the valve is presented. Section seven compares the results of the two models for several valve parameters and the last section validates the models by comparison with flow rate measurements on a valve prototype.

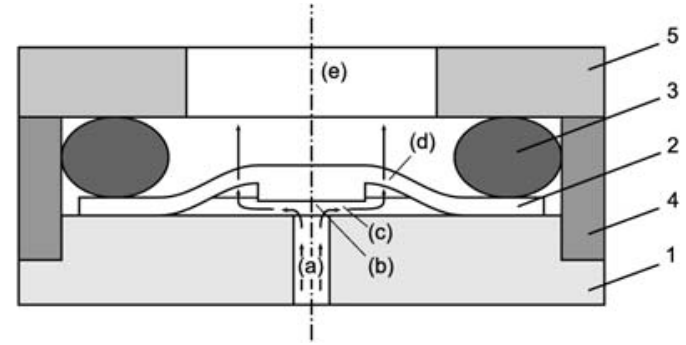

(a)

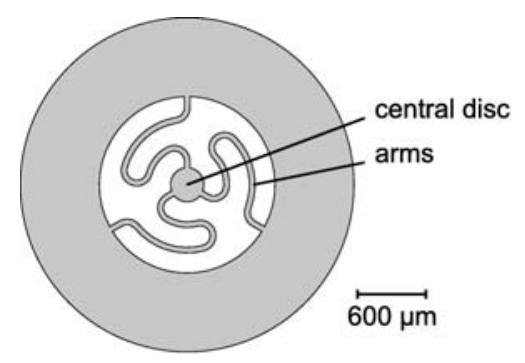

(b)

Fig. 1: Valve presentation (a) valve structure (scale is not respected); (b) spider-spring view.

\section{Valve Structure}

As shown on Fig. 1(a), the valve is composed of a seat (1), a spider-spring (2), an o-ring (3), an intermediate ring (4) and a lid (5). The principle of the spider-spring is explained in detail in [12]. The spider-spring is wedged between the seat and the lid, the o-ring guaranteeing a uniform force along the circumference of the spider-spring.

${ }^{\mathrm{a} C}$ Corresponding author; e-mail: olivier.smal@uclouvain.be, tel: +3210474796 , fax: +3210472501 
The extra thickness on the central disc of the spiderspring creates a pre-stress in the spring at rest position to avoid backflow [13]. The valve is represented in its open position, the fluid flowing upwards, passing between the 'arms' of the spider-spring (see Fig. 1(b)).

\section{Valve Manufacturing}

In our approach, we choose to manufacture the various parts of the valve separately. For the spider-spring, we focus on two different manufacturing techniques (Electro Discharge Machining [14, 15] - EDM - and SU8 lithography $[16,17])$ implying two different materials. The EDM milling procedure was chosen as it was one of the few mechanical manufacturing possibilities to realize the interior cuttings. Since this procedure can only work on a conducting material and since the part will have to work in water (as a valve component), we choose stainless steel as material. The second technique is SU8 lithography. This is a technique coming from the micro-electronic world and allowing to realize multi-layered components. Due to its quite simple processes and the relatively good mechanical properties, SU8 is used in many microfluidic applications. The detailed manufacturing procedure as well as manufacturing accuracy for these two methods are described in [11].

The other parts of the valve (i.e. the seat, the intermediate ring and the lid) are manufactured with a classic turning machine (except for the hole in the center of the seat which in made on a micro milling machine). All these parts are realized in aluminium.

\section{Description of the flow}

The flow is described in Fig. 1(a). The fluid flows first through a channel (a) of cylindrical cross section (diameter $=200 \mu \mathrm{m}$ ). After that the fluid is deviated when it hits the central disc of the spider-spring (b). Next the fluid flows through the small section (c) formed at the time of the opening of the valve, between the disc and the seat. Then, the fluid flows between the 'arms' of the spiderspring (d) and eventually flows through the outlet section (e) which is much larger than the inlet section. The flow in these regions can be characterized as follows:

- region (a) is a flow in a cylindrical channel,

- region (b) is a singular pressure drop due to the abrupt fluid flow deviation,

- region (c) is a flow between two parallel discs,

- region (d) is a pressure drop caused by the spiderspring's 'arms',

- region (e) is a flow in a cylindrical channel .

\section{$5 \quad$ Fluidic Analytical modelling}

This section first presents the simplifying modelling assumptions. Then the model is subdivided into four parts: a force analysis on the disc, the determination of the singular pressure losses when fluid deviates on the disc, the calculation of the flow rate and the initialization phase.

Here are the hypotheses taken into account:

- the valve is simplified as a simple disc on its seat (the effect of the 'arms' of the spider-spring on the fluid is neglected, i.e. term (d) in section 4),

- the central disc is non deformable,

- during its displacement, the central disc stays parallel to the seat,

- the disc is pressed onto its seat by a spring with a stiffness noted $k_{m}$ and a pre-stress force noted $F_{0}$,

- the flow is laminar in regions (a), (c) and (e) as shown on Fig. 1(a),

- the pressure drop in region (e) is neglected.

The main geometrical characteristics of the valve are presented in Fig. 2. The flow through the valve is studied for a given pressure difference between inlet and outlet. The vertical displacement of the disc is used as auxiliary variable.

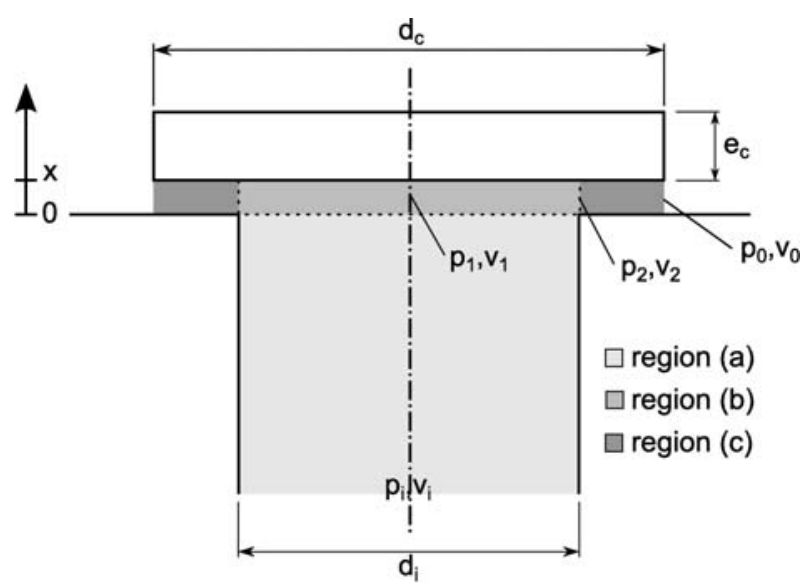

Fig. 2: Main geometrical characteristics and pressure distribution in the valve for open position.

\subsection{Force analysis}

For a given vertical displacement of the disc $x$, the return force on the disc exerted by the spring is

$$
F_{f}=F_{0}+k_{m} x
$$

where $F_{f}$ is the total force. For the disc to be in equilibrium, this force should be balanced by the force $\left(F_{p}\right)$ induced by pressures of the fluid on the disc. The surface 
of the disc is divided into two parts: the first corresponds to the inlet section $\left(A_{i}\right)$ and the second, the difference between the surface of the disc and $A_{i}$, will be noted $A_{s}$. These elements are illustrated on Fig. 3. We have immediately:

$$
\begin{aligned}
A_{i} & =\frac{\pi d_{i}{ }^{2}}{4} \\
A_{s} & =\frac{\pi d_{c}{ }^{2}}{4}-A_{i}=\pi \frac{d_{c}{ }^{2}-d_{i}{ }^{2}}{4}
\end{aligned}
$$

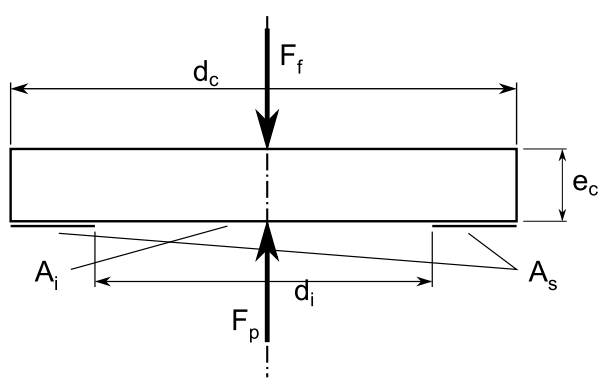

Fig. 3: Forces on the central disc

Let us define the pressures on the lower face of the disc as follows (see Fig. 2): $p_{1}$ on the center, $p_{2}$ at $d=d_{i}, p_{0}$ at $d=d_{c}$. On the upper face there is a uniform pressure $p_{0}$ (atmospheric pressure). So, the forces induced by the pressure of the fluid on the disc are:

$$
\begin{aligned}
F_{p}= & \int_{0}^{2 \pi} \int_{0}^{R_{1}}\left(\frac{p_{2}-p_{1}}{R_{1}{ }^{2}} r^{2}+p_{1}-p_{0}\right) r d r d \theta \\
& +\int_{0}^{2 \pi} \int_{R_{1}}^{R_{2}} \frac{p_{0}-p_{2}}{R_{2}-R_{1}}\left(r-R_{2}\right) r d r d \theta \\
= & \pi R_{1}^{2}\left(\frac{p_{2}+p_{1}}{2}-p_{0}\right)+ \\
& 2 \pi \frac{p_{2}-p_{0}}{R_{2}-R_{1}}\left(\frac{R_{2}{ }^{3}}{6}+\frac{R_{1}{ }^{3}}{3}-\frac{R_{2} R_{1}{ }^{2}}{2}\right),
\end{aligned}
$$

where $R_{1}=d_{i} / 2, R_{2}=d_{c} / 2$ and with this expression of $F_{p}$ we can obtain the value of $x$. Indeed, we have at equilibrium:

$$
F_{f}=F_{0}+k_{m} x=F_{p} .
$$

To determine the values of $p_{1}$ and $p_{2}$, we use the Bernoulli relation and we neglect the regular pressure losses in region (a) and (b). We have for region (a)

$$
\begin{aligned}
p_{1} & =p_{i}+\frac{\rho v_{i}^{2}}{2} \\
& =p_{i}+\frac{\rho}{2}\left(\frac{4 \dot{m}}{\rho \pi d_{i}{ }^{2}}\right)^{2},
\end{aligned}
$$

and for region (b):

$$
\begin{aligned}
p_{2} & =p_{1}-\frac{\rho v_{2}{ }^{2}}{2}-\zeta \frac{\rho v_{i}^{2}}{2} \\
& =p_{1}-\frac{\rho}{2}\left(\frac{\dot{m}}{\rho \pi d_{i} x}\right)^{2}-\zeta \frac{\rho}{2}\left(\frac{4 \dot{m}}{\rho \pi d_{i}{ }^{2}}\right)^{2} .
\end{aligned}
$$

In these two equations, $\dot{m}$ is the mass flow rate. In equation (7), the term containing $\zeta$ corresponds to the pressure drop resulting from the deviation of the flow on the disc. The expression of coefficient $\zeta$ can be found in [18].

\subsection{Flow rate}

The flow between the seat and the disc (Fig. 4(a) and region (c) in Fig. 2) is approximated as a laminar flow (last assumption of section 5) between two rectangular parallel plates (Fig. 4(b)). An analytical solution for the annular geometry could be obtained but this would only slightly improve the prediction quality.

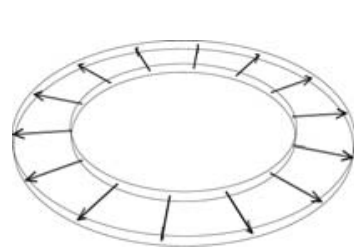

(a)

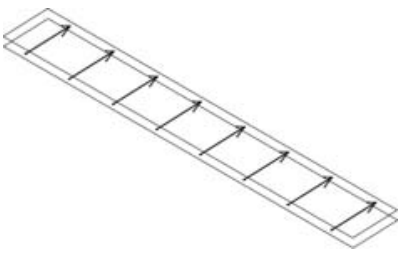

(b)
Fig. 4: Flow between the disc and the seat (a) real case; (b) approximation.

With this hypothesis, the mean velocity of the flow is

$$
u_{\text {mean }}=-\frac{d p}{d x} \frac{x^{2}}{12 \mu} .
$$

The flow rate through the small bypass section is simply

$$
\dot{Q}=\pi\left(\frac{d_{c}+d_{i}}{2}\right) x u_{\text {mean }}
$$

where the average speed is multiplied by the average bypass section which correspond to the lateral surface of a cylinder. From these equations we can obtain the mass flow rate

$$
\begin{aligned}
\dot{m} & =\dot{Q} \rho \\
& =\left(\pi \frac{d_{c}+d_{i}}{2}\right) \rho \frac{\Delta p}{\Delta x} \frac{x^{3}}{12 \mu},
\end{aligned}
$$

where $\Delta x$ is the distance covered by the fluid, which is half of the difference between the disc diameter and the inlet diameter

$$
\Delta x=\frac{d_{c}-d_{i}}{2} .
$$

So, the mass flow rate becomes:

$$
\dot{m}=\pi\left(d_{c}+d_{i}\right) \frac{\Delta p}{d_{c}-d_{i}} \frac{x^{3}}{12 \nu} .
$$

In our case, the pressure difference $\Delta p$ to be considered is defined in section 5.1.

$$
\dot{m}=\pi\left(d_{c}+d_{i}\right) \frac{p_{2}-p_{0}}{d_{c}-d_{i}} \frac{x^{3}}{12 \nu} .
$$


Combining this expression with equations (4) and (7), the flow rate is given by the solution of a quadratic equation of the form:

$$
a b \dot{m}^{2}+\dot{m}-a\left(p_{i}-p_{0}\right)=0,
$$

with the folowing definition for the two parameters:

$$
\begin{aligned}
a & =\pi \frac{d_{c}+d_{i}}{d_{c}-d_{i}} \frac{x^{3}}{12 \nu} \\
b & =(\zeta-1) \frac{\rho}{2}\left(\frac{4}{\pi d_{i}{ }^{2} \rho}\right)^{2}+\frac{\rho}{2}\left(\frac{1}{\pi d_{i} x \rho}\right)^{2} .
\end{aligned}
$$

\subsection{Solution}

The equations of the flow rate and of the vertical position of the disc have to be solved in an iterative way, since the mass flow rate $\dot{m}$ appears in the expression of $x$ (see section 5.1). For the initialization phase, the various pressure losses introduced in equations (6) and (7) are neglected so the expression of the vertical displacement of the disc is

$$
x=\frac{\left(A_{i}+A_{s} / 2\right)\left(p_{i}-p_{0}\right)-F_{0}}{k_{m}},
$$

and the mass flow rate becomes simply

$$
\dot{m}=\pi\left(d_{c}+d_{i}\right) \frac{p_{i}-p_{0}}{d_{c}-d_{i}} \frac{x^{3}}{12 \nu} .
$$

\section{Fluid-Structure FEM}

This section presents the finite-element model of the valve, which is based on fluid dynamics, for computing the motion of the fluid. The model is realized with COMSOL Multiphysics ${ }^{\circledR}$ using Incompressible Navier-Stokes and Moving Mesh (ALE) application modes. The fluid flow solution gives the forces exerted by the fluid on the domain boundaries. A force balance is then realized on the disc, leading to the new position of the disc, what modifies the domain and deforms the mesh. The hypotheses presented in section 5 are kept for this finite-element model. As the fluid flow moves up or down the disc, the simulation needs to be done in a deforming geometry, defined by the COMSOL Multiphysics Moving Mesh application mode. The force balance of the disc is calculated from an Ordinary Differential Equation (ODE).

\subsection{Model definition}

The model is set up in 2D axial symmetry (Fig. 5). The inlet tube has a diameter of $0.2 \mathrm{~mm}$ and the outlet tube a diameter of $0.6 \mathrm{~mm}$, the disc has a diameter of $0.15 \mathrm{~mm}$ and a thickness of $0.04 \mathrm{~mm}$ and the fluid is water (density $\rho=1000 \mathrm{~kg} / \mathrm{m}^{3}$, dynamic viscosity $\mu=1 \times 10^{-3} \mathrm{~Pa} . \mathrm{s}$ ). The system is driven by pressure difference: the outlet is maintained to zero, while the inlet is submitted to a ramp starting at $\mathrm{t}=0.2 \mathrm{~s}$ and reaching full pressure at $\mathrm{t}=0.4 \mathrm{~s}$.

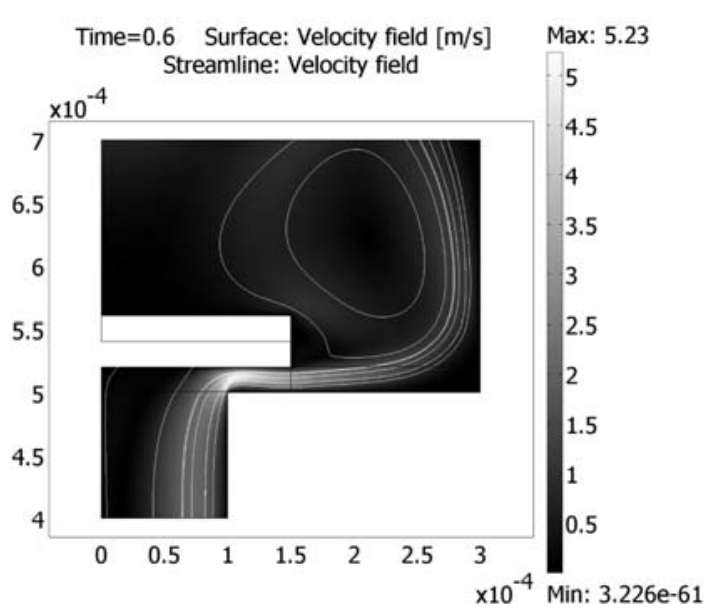

Fig. 5: The velocity field of the flow under an inlet pressure of 0.1 bar for a valve with a stiffness of $10 \mathrm{~N} / \mathrm{m}$ and a pre-stress force of $10 \times 10^{-5} \mathrm{~N}$.

\subsection{Domain equations}

The fluid flow is described by the Navier-Stokes equations:

$$
\begin{aligned}
& \rho \frac{\partial u}{\partial t}-\mu \cdot \nabla^{2} u+\rho(u \cdot \nabla) u+\nabla p=0 \\
& \nabla \cdot u=0
\end{aligned}
$$

where $\rho$ denotes the density $\left[\mathrm{kg} \mathrm{m}^{-3}\right], u$ the velocity vector $\left[m s^{-1}\right], \mu$ the dynamic viscosity $\left[N s m^{-2}\right]$, and $p$ the pressure $[\mathrm{Pa}]$. The equations are set up and solved in axial symmetry on a deformed mesh with the geometry presented on Fig. 5. The ODE that describes the force balance is:

$$
m \ddot{x}=F_{g}+F_{z}-F_{p r e}-k_{m} x
$$

where $m[k g]$ denotes the mass of the disc, $x[m]$ the position of the disc, $F_{g}[N]$ the gravitational force, $F_{z}[N]$ the $\mathrm{z}$ component of the force that the fluid exerts on the disc, $F_{\text {pre }}[N]$ the pre-stress force in the spring and $k_{m} x$ corresponds to the return force of the spring. The gravitational force will be neglected and in the axially symmetric model, the $F_{z}$ term will be:

$$
F_{z}=2 \pi \int_{S} r n\left(-p I+\mu\left(\nabla u+(\nabla u)^{T}\right)\right) d S
$$

where $r[m]$ is the radial coordinate and $n$ is the normal vector on the surface of the disc.

\subsection{Boundary conditions}

For the fluid simulation, the boundary condition at the inlet imposes a 'normal flow/pressure' and at the outlet a zero pressure. The moving mesh follows the displacement of the disc, which is the only moving part. The disc can only move along the $\mathrm{z}$-axis ( $\mathrm{r}$ displacement fixed to zero). 


\subsection{Mesh elements}

The finite element computations are only realized on the fluid passage section. The main parameters of the mesh presented on Fig. 6 are the followings: number of points $=1745$; number of triangular elements $=2980$; number of degrees of freedom $=31668$.

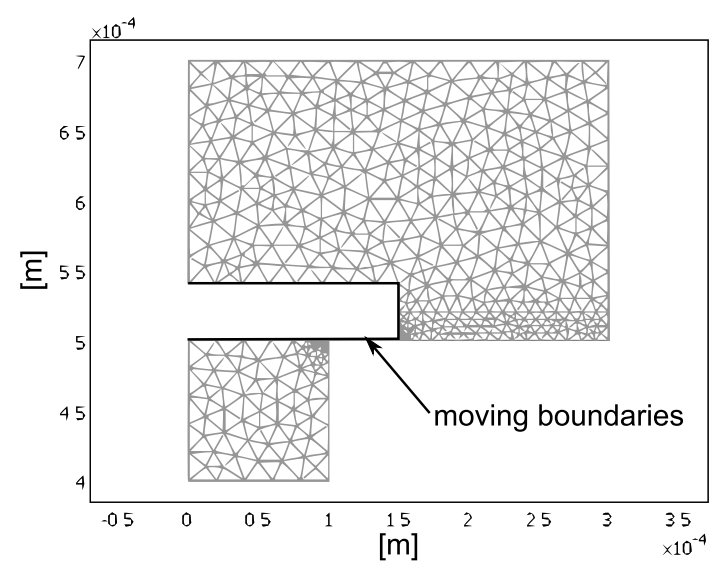

Fig. 6: The mesh of the fluid section of the valve.

\section{Comparison between models}

This part presents and compares the results of the two models for various values of stiffness and pre-stress of the ortho-planar spring of the valve. Table 1 presents the main parameters (as defined in Fig. 2, $d_{o}$ being the diameter of the outlet section) of the valves modelled. The results of the models, i.e. the values of the flow rate and the vertical position of the disc versus pressure difference, are presented in Fig. 7 (simulation time is 70 minutes for the FEM with COMSOL32 only for one valve, on a Pentium D $2.8 \mathrm{GHz}$ with $1.5 \mathrm{~Gb}$ Ram and $0.5 \mathrm{~s}$ for the analytical model). In this figure, model without $\zeta$ is a simplified version of our analytical model neglecting the pressure drop resulting from the deviation of the flow on the disc (term containing $\zeta$ in (7)).

Table 1: Parameters of the various valves

\begin{tabular}{lcrr}
\hline & & $\mathrm{a}$ & $\mathrm{b}$ \\
\hline$d_{c}$ & {$[\mathrm{~m}]$} & $300 \times 10^{-6}$ & $300 \times 10^{-6}$ \\
$e_{c}$ & {$[\mathrm{~m}]$} & $40 \times 10^{-6}$ & $40 \times 10^{-6}$ \\
$d_{i}$ & {$[\mathrm{~m}]$} & $200 \times 10^{-6}$ & $200 \times 10^{-6}$ \\
$d_{o}$ & {$[\mathrm{~m}]$} & $600 \times 10^{-6}$ & $600 \times 10^{-6}$ \\
$k_{m}$ & {$[\mathrm{~N} / \mathrm{m}]$} & 9.368 & 18.834 \\
$F_{0}$ & {$[\mathrm{~N}]$} & $93.68 \times 10^{-6}$ & $188.34 \times 10^{-6}$ \\
\hline
\end{tabular}

The Fig. 7 shows the good agreement of our analytical model with respect to the FEM. As the strategy of the analytical model is to determine first the forces on the disc and hence the vertical position of the central disc and secondly the flow rate for this opening of the valve, we plot the disc position and the flow rate versus the inlet pressure. The correspondence is quite good for the position and slightly less for the flow rate. This difference could be due to the approximation made for the pressure loss of the fluid deviation close to the disc.
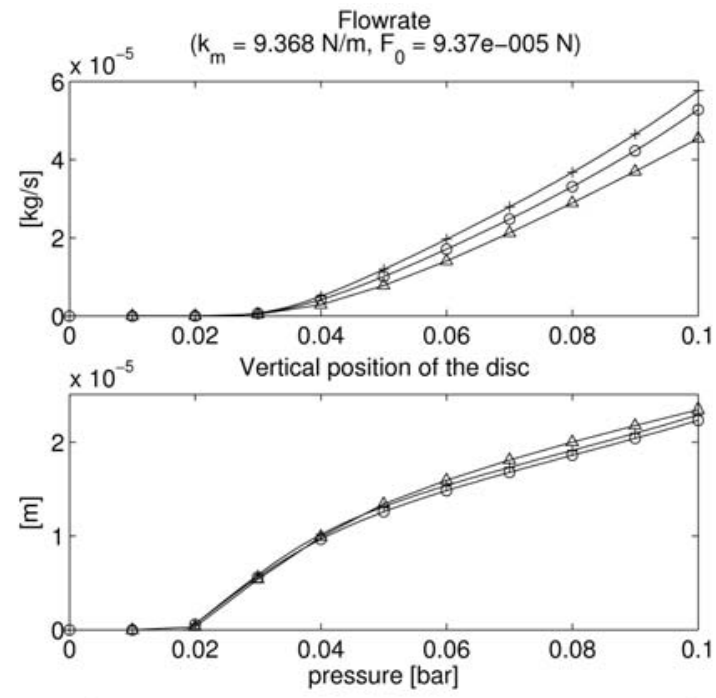

- analytical model + model without $\zeta \Delta$ FEM

(a)
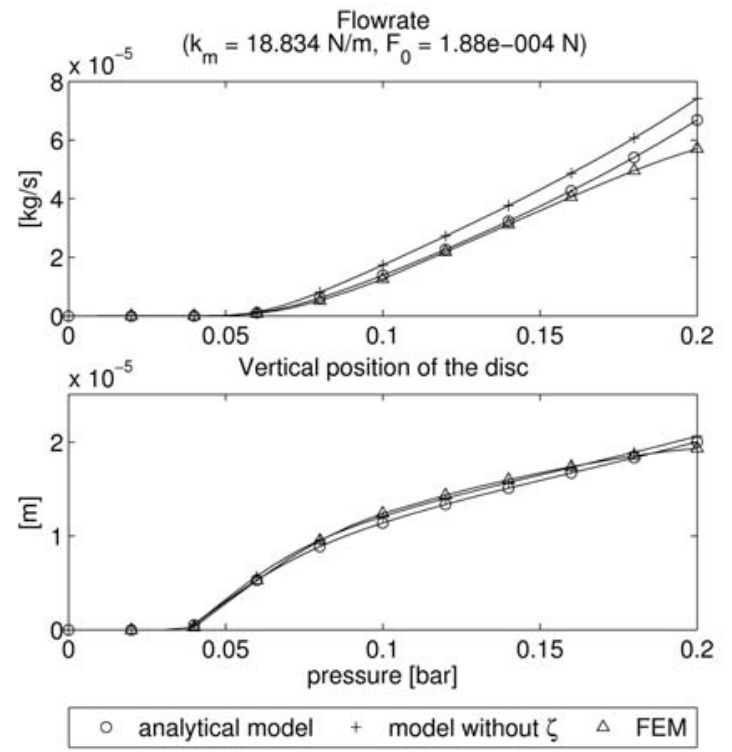

(b)

Fig. 7: Comparison of the models for two sets of parameters. The flowrate through the valve and the vertical position of the disc are plotted versus the inlet pressure.

\section{Validation of the models}

In this section, both the analytical and the finite-element models are compared to measurements made on valve prototypes which are realized as presented in section 3 . 
The realized test bed can be decribed as follows: a water tank is connected to the valve inlet while a graduated capillary is placed at the exit. The theoretical flow rate of the valve is compared to measurements of flow rate through the valve when varying the inlet pressure with a water column. The detailed experimental procedure is described in [13]. The dimensions of the valve and spider-spring prototype were accurately measured using an optical measurement machine (Mycrona Signum SL) and these dimensions were used both in the analytical and the finite-element models. Results are presented in Fig. 8 where analytical model is the model we developped in this paper, FEM is our finite-element model built with COMSOL Multiphysics ${ }^{\circledR}$, Oosterbroek model is an existing model [9] where the entrance and exit effects are neglected, simplifying the flow to a Poiseuille flow-profile over the full length of the gap and measurements are values from flow measurements on our valve prototypes. The various parameters (as defined in Fig. 2, $d_{o}$ being the diameter of the outlet section) of the measured valves are presented in table 2 .

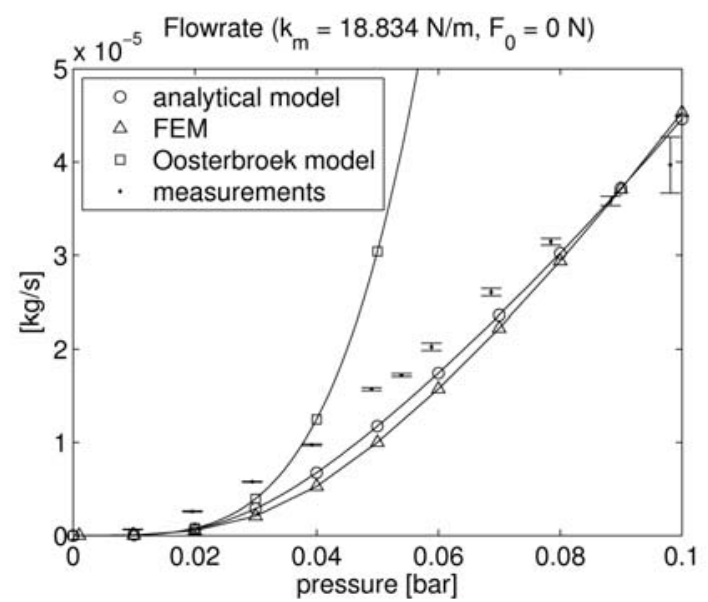

(a)

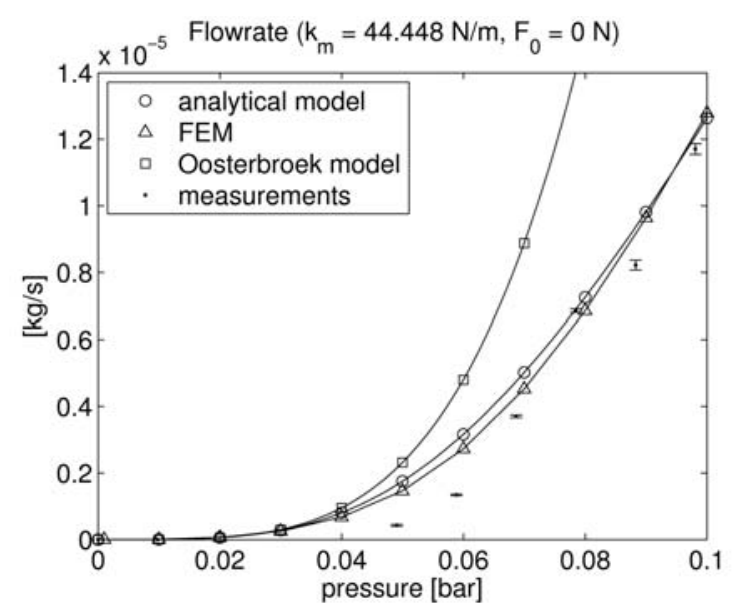

(b)

Fig. 8: Validation of the model for two sets of parameters. Graphs shows the flowrate through the valve versus the inlet pressure.
Fig. 8 shows the good correspondence of our models with the measurements made on the valve. Concerning the Oosterbroek model, we see a divergence from the other curves as soon as the flow rate becomes larger. For these higher flow rates, the entrance and exit effects may no longer be neglected. This point had already been discussed in [9]. Coming back to our analytical model and our FEM, the small differences with measurements could be explained by the hypotheses we made in section 5 . The major neglected terms in our analytical model are:

- the pressure drop due to the arms of the spiderspring

- the deformation of the central disc

- the pressure drop due to the tubing existing in our test bed.

Table 2: Parameters of the various valves.

\begin{tabular}{ccrr}
\hline & & $\mathrm{a}$ & $\mathrm{b}$ \\
\hline$d_{c}$ & {$[\mathrm{~m}]$} & $300 \times 10^{-6}$ & $300 \times 10^{-6}$ \\
$e_{c}$ & {$[\mathrm{~m}]$} & $40 \times 10^{-6}$ & $40 \times 10^{-6}$ \\
$d_{i}$ & {$[\mathrm{~m}]$} & $230 \times 10^{-6}$ & $230 \times 10^{-6}$ \\
$d_{o}$ & {$[\mathrm{~m}]$} & $600 \times 10^{-6}$ & $600 \times 10^{-6}$ \\
$k_{m}$ & {$[\mathrm{~N} / \mathrm{m}]$} & 18.834 & 44.448 \\
$F_{0}$ & {$[\mathrm{~N}]$} & $0 \times 10^{-6}$ & $0 \times 10^{-6}$ \\
\hline
\end{tabular}

\section{Conclusion}

The fluid flow analytical model presented in this paper is in good agreement with a finite-element model developed using COMSOL Multiphysics. A good correspondence for the position of the disc as well as for the flow rate is observed. The analytical model is then validated by flow rate measurements made on a valve prototype. The main advantage of this analytical model is its time consumption $(0.5 \mathrm{~s}$ with respect to $70 \mathrm{~min}$ for the FEM as presented in section 7). A second advantage is that this analytical expression can be used for optimization of the valve. If the behavior of the valve is well simulated, we can study the influence of several parameters (for example the diameter of the disc, the stiffness of the spring, the inlet diameter channel...) and optimize the valve for a specific application. It can also be used to simulate the behavior of a micropump, if models for the other constitutive parts of the pump are added.

\section{References}

[1] D. J. Laser, J. G. Santiago, A review of micropumps, Journal of Micromechanics and Microengineering 14 (2004) R35-R64. 
[2] B. Li, Q. Chen, D.-G. Lee, J. Woolman, G. P. Carman, Development of large flow rate, robust, passive micro check valves for compact piezoelectrically actuated pumps, Sensors and Actuators A 117 (2005) 325-330.

[3] N.-T. Nguyen, T.-Q. Truong, K.-K. Wong, S.-S. Ho, C. L.-N. Low, Micro check valves for integration into polymeric microfluidic devices, Journal of Micromechanics and Microengineering 14 (2004) 6975.

[4] X. Yang, C. Grosjean, Y.-C. Tai, C.-M. Ho, A mems thermopneumatic silicone rubber membrane valve, Sensors and Actuators A 64 (1998) 101-108.

[5] N.-T. Nguyen, T.-Q. Truong, A fully polymeric micropump with piezoelectric actuator, Sensors and Actuators B 97 (2004) 137-143.

[6] B. Wang, X. Chu, E. Li, L. Li, Simulations and analysis of a piezoelectric micropump, Ultrasonics 44 (2006) e643-e646.

[7] T. C. Yih, C. Wei, B. Hammad, Modeling and characterization of a nanoliter drug-delivery mems micropump with circular bossed membrane, Nanomedicine 1 (2005) 164-175.

[8] Q. Gong, Z. Zhou, Y. Yang, X. Wang, Design, optimization and simulation on microelectromagnetic pump, Sensors and Actuators 83 (2000) 200-207.

[9] R. Oosterbroek, S. Schlautmann, J. Berenschot, T. Lammerink, A. van den Berg, M. Elwenspoek, Modeling and validation of flow-structure interactions in passive micro valves, in: Technical proceedings of the 1998 International Conference on Modeling and Simulation of Microsystems, 1998, pp. 528-533.

[10] J. J. Parise, L. L. Howell, S. P. Magleby, Orthoplanar linear-motion springs, Mechanism and Machine Theory 36 (2001) 1281-1299.

[11] O. Smal, B. Dehez, B. Raucent, M. De Volder, J. Peirs, D. Reynaerts, F. Ceyssens, J. Coosemans,
R. Puers, Modelling, characterization and testing of an ortho-planar micro-valve, Journal of Micro Nano Mechatronics 4 (3) (2008) 131-143.

[12] O. Smal, B. Dehez, B. Raucent, M. De Volder, J. Peirs, D. Reynaerts, F. Ceyssens, J. Coosemans, R. Puers, Modelling and characterisation of an ortho-planar micro-valve, in: S. Ratchev (Ed.), Proceedings of the IFIP TC5 WG5.5 Third International Precision Assembly Seminar (IPAS '2006), Springer, Bad Hofgastein, Austria, 2006, pp. 315326.

[13] O. Smal, B. Raucent, F. Ceyssens, R. Puers, M. De Volder, D. Reynaerts, Design and testing of an ortho-planar micro-valve, in: S. Ratchev (Ed.), Proceedings of the IFIP TC5 WG5.5 Fourth International Precision Assembly Seminar (IPAS '2008), Springer, Chamonix, France, 2008, pp. 75-86.

[14] W. Meeusen, Micro Electro-Discharge Machining: Technology, Computer-Aided Design \& Manufacturing and applications, Phd Thesis, KULeuven, Belgium, 2003

[15] X. Song, D. Reynaerts, W. Meeusen, H. Van Brussel, Investigation of Micro-EDM for Silicon Microstructure Fabrication, In Proceedings of SPIE Symposium on Micromachining and Microfabrication, 729-799, 1999.

[16] H. Lorenz, M. Despont, N. Fahrni, N. LaBianca, P. Renaud, P. Vettiger, SU-8: a low-cost negative resist for MEMS, Journal of Micromechanics and Microengineering, 7, 121-124, 1997

[17] R. Feng, R.J. Farris, Influence of processing conditions on the thermal and mechanical properties of SU8 negative photoresist coatings, Journal of Micromechanics and Microengineering, 13, 80-88, 2003

[18] I. E. Idel'cik, Mémento des pertes de charges, 3rd Edition, Eyrolles, 1986. 